Теорія Ймовір. та Матем. Статист. Вип. 75, 2006
Theor. Probability and Math. Statist.

No. 75, 2007, Pages 161-165 S 0094-9000(08)00722-9

Article electronically published on January 25, 2008

\title{
PARAMETER-DEPENDENT INTEGRALS WITH RESPECT TO GENERAL RANDOM MEASURES
}

UDC 519.21

\author{
V. M. RADCHENKO
}

\begin{abstract}
We study integrals of real functions considered with respect to general random measures. The integrals are assumed to depend on a parameter. We obtain sufficient conditions for the existence of a continuous version of random functions and sufficient conditions such that a random measure generated by increments of these random functions exists.
\end{abstract}

\section{INTRODUCTION}

Let $\mathbf{X}$ be an arbitrary set, $\mathcal{B}$ a $\sigma$-algebra of subsets of $\mathbf{X},(\Omega, \mathcal{F}, \mathrm{P})$ a complete probability space. By $L_{0}=L_{0}(\Omega, \mathcal{F}, \mathrm{P})$ we denote the set of all random variables (more precisely, the set of classes of $\mathrm{P}$-equivalent random variables). Convergence in $L_{0}$ means convergence in probability.

Definition. A $\sigma$-additive mapping

$$
\mu: \mathcal{B} \rightarrow L_{0}
$$

is called a random measure on $\mathcal{B}$.

We do not assume that $\mu$ is nonnegative or that it is adapted to a certain filtration. In this sense, our definition is general. Throughout the paper, the symbol $\mu$ stands for a random measure.

First we consider some examples. If $X(x), 0 \leq x \leq b$, is a continuous square integrable martingale, then $\mu(A)=\int_{0}^{b} I_{A}(x) d X(x)$ is a random measure on Borel subsets of the interval $[0, b]$. The integral with respect to the fractional Brownian motion $B^{H}(x)$ with Hurst index $H>\frac{1}{2}$ also defines a random measure (this follows from inequality (1.5) of [1]). Other examples and sufficient conditions for increments of a stochastic process to generate a random measure can be found in Chapters 7 and 8 of [2].

An integral of the form $\int_{A} h(x) d \mu(x)$ is constructed and studied in [3] where $h: \mathbf{X} \rightarrow \mathbb{R}$ is a measurable real function and $A \in \mathcal{B}$. The construction of this integral is standard and uses an approximation by simple functions. A similar construction is used in Chapter 7 of [2] (also see [4]). In particular, every measurable function $h$ is integrable with respect to any random function $\mu$. An analogue of the Lebesgue dominated convergence theorem holds for this integral (see Corollary 1.2 in [3] or Proposition 7.1.1 in [2]).

Let $\mathbf{T}$ be an arbitrary set and let a function $f: \mathbf{T} \times \mathbf{X} \rightarrow \mathbb{R}$ be integrable in the variable $x$ and with respect to measure $\mu$ for all fixed $t \in \mathbf{T}$. One can consider the

2000 Mathematics Subject Classification. Primary 60G57.

Key words and phrases. Random measure, stochastic integral dependent on a parameter, continuity of sample paths of a stochastic process. 
random function

$$
\eta(t)=\int_{\mathbf{X}} f(t, x) d \mu(x), \quad t \in \mathbf{T} .
$$

The spectral representation of a wide sense stationary stochastic process $\eta(t)$ is an example of the latter representation (with an obvious generalization to the case of a complexvalued function $f$ ).

Conditions for the continuity of sample paths and for some other properties of stochastic processes $\eta(t)$ of the form (1) are obtained in this paper.

\section{The CONTINUity OF TRAJECTORIES OF AN INTEGRAL}

Theorem 1. Let $\mathbf{X}=[a, b] \subset \mathbb{R}$, let $\mathcal{B}$ be the $\sigma$-algebra of Borel subsets of $[a, b]$, and let $\mathbf{T}$ be a metric space. Assume that a function $f(\cdot, x)$ is continuous on $\mathbf{T}$ for all $x \in[a, b]$ and

$$
|f(t, x)-f(t, y)| \leq C|x-y|^{\gamma}
$$

for some $\gamma>\frac{1}{2}, C>0$, and all $t \in \mathbf{T}$ and $x, y \in[a, b]$. Then the random function $\eta(t)$ defined by (1) possesses a version whose trajectories are continuous on $\mathbf{T}$.

Proof. For all $n \geq 0$, put

$$
d_{k n}=a+\frac{k}{2^{n}}(b-a), \quad 0 \leq k \leq 2^{n}, \quad \Delta_{k n}=\left(d_{(k-1) n}, d_{k n}\right], \quad 1 \leq k \leq 2^{n} .
$$

Consider the functions

$$
f_{n}(t, x)=f(t, a) I_{\{a\}}(x)+\sum_{1 \leq k \leq 2^{n}} f\left(t, d_{(k-1) n}\right) I_{\Delta_{k n}}(x) .
$$

Condition (2) implies, in particular, that $f(t, \cdot)$ is continuous; thus $f_{n}(t, x) \rightarrow f(t, x)$ for all $t, x$. An analogue of the Lebesgue theorem (Corollary 1.2 of [3]) implies that

$$
\int_{[a, b]} f_{n}(t, x) d \mu(x) \stackrel{\mathrm{P}}{\rightarrow} \int_{[a, b]} f(t, x) d \mu(x), \quad n \rightarrow \infty
$$

for all $t$. Therefore

$$
\int_{[a, b]} f_{n}(t, x) d \mu(x)=f(t, a) \mu(\{a\})+\sum_{1 \leq k \leq 2^{n}} f\left(t, d_{(k-1) n}\right) \mu\left(\Delta_{k n}\right) \quad \text { a.s. }
$$

(Here and in what follows the abbreviation "a.s." means "almost surely".) Since $f(\cdot, x)$ is continuous, the right hand side of (5D) is a continuous function with respect to $t$ if $\omega \in \Omega$ is fixed. Thus it is sufficient to prove that the convergence of functions on the right hand side of (5) is almost surely uniform as $n \rightarrow \infty$.

Lemma 2 of 5 plays a key role in the proof. We have

$$
\sum_{n=0}^{\infty} 2^{-n \gamma} \sum_{k=1}^{2^{n}}\left|\mu\left(\Delta_{k n}\right)\right|<\infty \quad \text { a.s. }
$$

for all $[a, b] \subset \mathbb{R}, \gamma>\frac{1}{2}$, and $\mu$. 
Using inequality (2) and representation (5), consider the series

$$
\begin{aligned}
& \sum_{n \geq m}\left|\int_{[a, b]} f_{n}(t, x) d \mu(x)-\int_{[a, b]} f_{n-1}(t, x) d \mu(x)\right| \\
& \quad \leq \sum_{n \geq m} \sum_{1 \leq k \leq 2^{n}}\left|f\left(t, d_{(k-1) n}\right)-f\left(t, d_{\left(k^{\prime}-1\right)(n-1)}\right)\right|\left|\mu\left(\Delta_{k n}\right)\right| \\
& \quad \leq C(b-a)^{\gamma} \sum_{n \geq m} \sum_{1 \leq k \leq 2^{n}} 2^{-n \gamma}\left|\mu\left(\Delta_{k n}\right)\right|
\end{aligned}
$$

for an arbitrary $m \geq 0$. The number $k^{\prime}$ is chosen such that $\Delta_{k n} \subset \Delta_{k^{\prime}(n-1)}$. The latter sum converges to zero as $m \rightarrow \infty$ according to (6). Moreover the convergence is uniform with respect to $t \in \mathbf{T}$; thus (4) implies that the limit is a continuous version of $\eta(t)$.

Further we consider the question on the continuity of sample paths of integrals of real functions considered with respect to random measures. The continuity is studied with respect to the upper limit of integrals. An example of the constant function being equal to 1 everywhere shows that if one studies a rather wide class of integrands, then one has to assume that $\mu$ is such that continuous sample paths of the integral exist.

Theorem 2. Let $\mathbf{X}=[a, b] \subset \mathbb{R}$, let $\mathcal{B}$ be the $\sigma$-algebra of Borel subsets of $[a, b]$, and let the stochastic process $\mu(x)=\mu([a, x]), a \leq x \leq b$, possess a continuous version. Assume that a function $h:[a, b] \rightarrow \mathbb{R}$ is such that

$$
|h(x)-h(y)| \leq C|x-y|^{\gamma}
$$

for some $\gamma>\frac{1}{2}$ and $C>0$ and for all $x, y \in[a, b]$. Then the random function

$$
\zeta(t)=\int_{[a, t]} h(x) \mu(d x), \quad a \leq t \leq b,
$$

possesses a version whose trajectories are continuous on $[a, b]$.

Proof. We again use notation (3) on the interval $[a, b]$. The Hölder condition (8) implies that $h$ is continuous and bounded. Using an analogue of the Lebesgue theorem, we get

$$
\begin{gathered}
h(a) \mu(\{a\})+\sum_{k=1}^{2^{n}} h\left(d_{(k-1) n}\right) \mu\left(\left(d_{(k-1) n} \wedge t, d_{k n} \wedge t\right]\right) \stackrel{\mathrm{P}}{\rightarrow} \int_{[a, t]} h(x) \mu(d x), \\
n \rightarrow \infty,
\end{gathered}
$$

for every fixed $t$. Consider

$$
\zeta_{n}(t)=h(a) \mu(\{a\})+\sum_{k=1}^{2^{n}} h\left(d_{(k-1) n}\right) \mu\left(\left(d_{(k-1) n} \wedge t, d_{k n} \wedge t\right]\right)
$$

for a continuous version of $\mu(t)$. The stochastic process $\zeta_{n}$ possesses a continuous sample path for every fixed $\omega$. To complete the proof of the theorem, it is sufficient to show that the $\zeta_{n}(t)$ converge almost surely and uniformly on $[a, b]$. The limit is a version of $\zeta(t)$ 
according to (9). Similarly to (7) we have

$$
\begin{aligned}
& \sup _{a \leq t \leq b}\left|\zeta_{n}(t)-\zeta_{n}(t)\right| \\
& \leq \sup _{a \leq t \leq b} \sum_{i=m+1}^{n} \sum_{k=1}^{2^{i}}\left|h\left(d_{(k-1) i}\right)-h\left(d_{k^{\prime}(i-1)}\right)\right|\left|\mu\left(\left(d_{(k-1) i} \wedge t, d_{k i} \wedge t\right]\right)\right| \\
& \leq C(b-a)^{\gamma}\left(\sum_{i=m+1}^{n} \sum_{k=1}^{2^{i}} 2^{-i \gamma}\left|\mu\left(\Delta_{k i}\right)\right|\right. \\
& \left.\quad+\sup _{a \leq t \leq b} \sum_{m+1 \leq i \leq n, k: t \in\left(d_{(k-1) i}, d_{k i}\right]} 2^{-i \gamma}\left|\mu\left(\left(d_{(k-1) i} \wedge t, d_{k i} \wedge t\right]\right)\right|\right)
\end{aligned}
$$

for all $m<n$. The number $k^{\prime}$ is chosen such that $\Delta_{k n} \subset \Delta_{k^{\prime}(n-1)}$. We consider separately the terms for which $t$ belongs to the semiinterval $\Delta_{k i}$. For $n+1 \leq i \leq m$, we consider all $\left|\mu\left(\Delta_{k i}\right)\right|$ (this implies that the sum increases). Since the sample paths of $\mu(t)$ are continuous, they are bounded. Thus

$$
\left|\mu\left(\left(d_{(k-1) i} \wedge t, d_{k i} \wedge t\right]\right)\right| \leq M(\omega)
$$

for some random constant $M(\omega)$ and for all $t$. Taking into account (6), we prove the almost sure convergence to zero as $m, n \rightarrow \infty$ in (10).

\section{Conditions for An integral to Generate A RANDOM MEASure}

In this section, we consider general $\mathbf{X}$ and $\mathcal{B}$ and impose conditions on $\mathbf{T}$.

Theorem 3. Let $\mathbf{T}=[a, b] \subset \mathbb{R}$ and let

(i) $f(t, \cdot): \mathbf{X} \rightarrow \mathbb{R}$ be integrable with respect to $\mu$ for every $t \in[a, b]$,

(ii) $f(\cdot, x):[a, b] \rightarrow \mathbb{R}$ be an absolute continuous function on $[a, b]$ for every $x \in \mathbf{X}$,

(iii) the function $g(x)$ defined as the variation of $f(\cdot, x)$ on $[a, b]$ be integrable with respect to $\mu$.

Assume that a random function $\eta(t)$ is defined by (11). Then the random function of sets $\mu_{\eta}((s, t])=\eta(t)-\eta(s)$ can be extended to a random measure on the $\sigma$-algebra $\mathcal{B}$ of Borel subsets of $[a, b]$.

Proof. Since $f(\cdot, x)$ is absolutely continuous, it almost everywhere has the derivative for which the Newton-Leibniz formula holds. Moreover

$$
\int_{[a, b]}\left|\frac{\partial f(t, x)}{\partial t}\right| d t=g(x)
$$

(see, for example, Theorem IX.4.8 in [6]). Put

$$
\mu_{\eta}(A)=\int_{\mathbf{X}} d \mu(x) \int_{A} \frac{\partial f(t, x)}{\partial t} d t, \quad A \in \mathcal{B} .
$$

The $\sigma$-additivity of $\mu_{\eta}$ follows from (11) and an analogue of the Lebesgue dominated convergence theorem (Corollary 1.2 of [3]).

Integrals with respect to $\mu_{\eta}$ can be calculated effectively under some extra assumptions. Let a function $s:[a, b] \rightarrow \mathbb{R}$ be bounded and measurable. Then using a uniform approximation of $s$ by simple functions, one can prove that

$$
\int_{[a, b]} s(t) d \mu_{\eta}(t)=\int_{\mathbf{X}} d \mu(x) \int_{[a, b]} s(t) \frac{\partial f(t, x)}{\partial t} d t .
$$


Theorem 1 of 5 implies that if $\partial f / \partial t$ is bounded, then the latter integral equals the integral on $[a, b] \times \mathbf{X}$ with respect to the product of measures $d t \times d \mu(x)$. The iterated integral written in the different order is well defined according to Theorem 2 of $[5]$.

\section{BIBLIOGRAPHY}

1. J. Memin, Yu. Mishura, and E. Valkeila, Inequalities for the moments of Wiener integrals with respect to a fractional Brownian motion, Statist. Probab. Lett. 57 (2001), no. 2, 197-206. MR 1822771 (2002b:60096)

2. S. Kwapień and W. A. Woyczyński, Random Series and Stochastic Integrals: Single and Multiple, Birkhäuser, Boston, 1992. MR1167198 (94k:60074)

3. V. N. Radchenko, Integrals with respect to general random measures, Proceedings of the Institute of Mathematics, National Academy of Sciences of Ukraine, vol. 27, Institute of Mathematics, Kiev, 1999. (Russian)

4. V. N. Radchenko, Integrals with respect to random measures and random linear functionals, Teor. Veroyatnost. i Primenen. 36 (1991), no. 3, 594-596; English transl. in Theory Probab. Appl. 36 (1991), no. 3, 621-623. MR1141138 (93e:60093)

5. V. N. Radchenko, On the product of random and real measures, Teor. Imovir. Mat. Stat. 70 (2004), 144-148; English transl. in Theory Probab. Math. Statist. 70 (2005), 161-166. MR:2110872 (2005h:60146)

6. I. P. Natanson, Theory of Functions of a Real Variable, Third edition, "Nauka", Moscow, 1974; English transl., Frederick Ungar Publishing, New York, 1967. MR0354979 (50:7456)

Department of Mathematical Analysis, Faculty for Mechanics and Mathematics, National Taras Shevchenko University, Academician Glushkov Avenue, 6, Kyiv 03127, Ukraine

E-mail address: vradchenko@univ.kiev.ua

Received 20/JAN/2006

Translated by O. I. KLESOV 